\title{
Filmes de disseleneto de cobre e índio depositados por spray-pirólise
}

\section{(Copper indium diselenide films deposited by spray-pyrolysis)}

\author{
C.S.Manhanini, H.R. Paes Jr. \\ Laboratório de Materiais Avançados/CCT, Universidade Estadual do Norte Fluminense Darcy Ribeiro, \\ Av. Alberto Lamego, 2000, Campos dos Goytacazes, RJ \\ carlamanhanini@gmail.com,hervalpaes@gmail.com
}

\begin{abstract}
Resumo
Filmes de $\mathrm{Cu}_{1-\mathrm{x}} \operatorname{In}_{\mathrm{x}} \mathrm{Se}_{2}(0,45 \leq \mathrm{x} \leq 0,80)$ foram depositados em substrato de vidro pela técnica de spray-pirólise para utilização como camada absorvedora de células fotovoltaicas. As propriedades estruturais, morfológicas, ópticas e elétricas dos filmes preparados foram analisadas em função da variação da estequiometria utilizada. A análise por difração de raios X mostrou que os picos mais intensos foram de orientação (204/220) e que os filmes possuíram as fases CuSe, $\mathrm{CuSe}_{2}$ e CuInSe ${ }_{2}$. Os filmes apresentaram superfície uniforme e sem trincas, independente da estequiometria utilizada. Na caracterização elétrica, os filmes depositados apresentaram energia de ativação do processo de condução elétrica com valor médio de $0,74 \mathrm{eV}$ e comportamento típico para semicondutores. A caracterização óptica foi realizada no intervalo de comprimento de onda de 350 a $1100 \mathrm{~nm}$ e os filmes apresentaram coeficiente de absorção na ordem de $10^{3} \mathrm{~cm}^{-1}$ no comprimento de onda de $550 \mathrm{~nm}$ e gap óptico de $1,4 \mathrm{eV}$. Os resultados indicaram que a condição de deposição mais adequada para filmes visando sua aplicação como camada absorvedora teve como temperatura de substrato de $400{ }^{\circ} \mathrm{C}$, fluxo de solução de $1 \mathrm{~mL} / \mathrm{min}$, tempo de deposição de $10 \mathrm{~min}$ e estequiometria de $\mathrm{Cu}_{0,2} \mathrm{In}_{0,8} \mathrm{Se}_{2}$, obtendo assim filmes sem trincas, apresentando maior coeficiente de absorção de $6,8 \times 10^{3} \mathrm{~cm}^{-1}$ para o comprimento de onda de $550 \mathrm{~nm}$, espessura de aproximadamente $2,5 \mu \mathrm{m}$ e resistividade elétrica de $0,13 \mathrm{k} \Omega . \mathrm{m}$ na temperatura ambiente.
\end{abstract}

Palavras-chave: spray-pirólise, camada absorvedora, disseleneto de cobre e índio.

\begin{abstract}
$C u_{1-x} \operatorname{In}_{x} \mathrm{Se}_{2}(0.45 \leq x \leq 0.80)$ films were deposited on glass substrate by spray pyrolysis technique, for use as absorbing layer of photovoltaic cells. The structural, morphological, optical and electrical properties of the films were analyzed according to the variation of the stoichiometry used. The analysis by X-ray diffraction showed that the most intense peaks were of orientation (204/220) and the films have the phases CuSe, CuSe ${ }_{2}$ and CuInSe. The films showed uniform surface without cracks independently of the stoichiometry used. In the electrical characterization, the deposited films showed activation energy of the electrical conduction process with average value of $0.74 \mathrm{eV}$ and typical behavior for semiconductors. The optical characterization was performed at the wavelength gap of 350 to $1100 \mathrm{~nm}$, and the films showed absorption coefficient on the order of $10^{3} \mathrm{~cm}^{-1}$ in the wavelength of $550 \mathrm{~nm}$ and optical band gap of $1.4 \mathrm{eV}$. The results indicated that the most suitable condition for deposition of films for their application as absorbing layer had as substrate temperature $400^{\circ} \mathrm{C}$, a solution flow rate of $1 \mathrm{~mL} / \mathrm{min}$, deposition time of 10 min and stoichiometry of $\mathrm{Cu}_{0.2} \mathrm{In}_{0.8} \mathrm{Se} e_{2}$, thus obtaining films without cracks, with large absorption coefficient of $6.8 \times 10^{3} \mathrm{~cm}^{-1}$ for the wavelength of $550 \mathrm{~nm}$, thickness of approximately $2.5 \mu \mathrm{m}$ and electrical resistivity of $0.13 \mathrm{k} \Omega . \mathrm{m}$ at room temperature.
\end{abstract}

Keywords: spray-pyrolysis, absorbing layer, copper indium diselenide.

\section{INTRODUÇÃO}

A década de 90 marcou o desenvolvimento acelerado da indústria fotovoltaica. Visando ampliar os horizontes para utilização em massa da energia solar como opção energética, inúmeros programas mundiais foram lançados para a demonstração da viabilidade técnica-comercial da energia solar fotovoltaica em projetos de eletrificação rural nos países em desenvolvimento. Os maiores desafios que o setor enfrenta são a redução de custos dos sistemas fotovoltaicos e a eficiência de conversão. As novas tecnologias em desenvolvimento, principalmente a de filmes, reduzem significativamente os custos dos módulos fotovoltaicos, já que possibilitam a utilização de um volume menor de material para sua fabricação.

A estrutura cristalina do disseleneto de cobre e índio pertence à família das calcopiritas com estrutura tetragonal. Os compostos calcopiríticos são completamente miscíveis entre si, quimicamente estáveis e variando-se as proporções desses elementos obtêm-se propriedades semicondutoras, que absorvem intensamente a radiação solar [1, 2]. Disseleneto de cobre e índio (CIS), devido a seu gap óptico de 1,04 a $1,5 \mathrm{eV}$, alto coeficiente de absorção na faixa de $10^{5}$ $\mathrm{cm}^{-1}$, boa estabilidade térmica e elétrica, é um dos materiais semicondutores mais promissores utilizados em células solares nos últimos anos sendo utilizado como uma camada absorvedora na forma de filmes. Células solares baseadas em $\mathrm{CuInSe}_{2}$ já demonstraram eficiência de $19,9 \%$ para o 
dispositivo de uma única junção [2,3].

O semicondutor de composto ternário do grupo I-III$\mathrm{VI}_{2}$, o disseleneto de cobre e índio, vem se destacando perante os demais semicondutores no contexto de se obter elevada eficiência de conversão. O CIS também pode ser um semicondutor do tipo $\mathrm{n}$ ou $\mathrm{p}$, dependendo da estequiometria utilizada, tornando-o atrativo para aplicação em conversão fotovoltaica [2-4]. No entanto, a camada de absorção CIS da célula solar de alta eficiência é usualmente preparada por processos de deposição que utilizam vácuo, possuindo várias limitações, tais como o elevado custo de produção, complexidade do processo e dificuldade de produção em série. A técnica de spray-pirólise, que foi utilizada neste trabalho, possui vantagens em relação às demais, já que é de baixo custo e não utiliza vácuo durante a deposição. Assim, este trabalho teve como objetivos a preparação de filmes de disseleneto de cobre e índio sobre substrato de vidro por spray-pirólise e o estudo da influência das condições de deposição, processamento térmico e estequiometria sobre suas propriedades morfológicas, elétricas, ópticas e estruturais.

\section{EXPERIMENTAL}

A técnica spray-pirólise foi utilizada para a deposição dos filmes. Consistiu na incidência de um spray formado por um gás de arraste contendo uma solução precursora composta por íons de interesse. Este spray incidiu sobre substratos posicionados em uma chapa previamente aquecida. $\mathrm{O}$ spray em contato com o substrato aquecido propiciou uma pirólise levando à formação de vários compostos. Os filmes de disseleneto de cobre e índio foram depositados utilizando substratos de vidro. No processo de deposição, o fluxo do ar comprimido foi fixado em $1,5 \mathrm{kgf} / \mathrm{cm}^{2}$, fluxo da solução precursora em $1 \mathrm{~mL} / \mathrm{min}$, temperatura de substrato de $400{ }^{\circ} \mathrm{C}$ e tempo de deposição de 10 min. O conteúdo de cobre e índio nos filmes variou em proporção de 1-x para cobre e x para índio, sendo os valores de $x$ entre 0,40 e 0,80 . A solução precursora para a produção de filmes $\mathrm{Cu}_{1-x} \operatorname{In}_{x} \mathrm{Se}_{2}$ foi obtida a partir da mistura de água deionizada com sais de cloreto de cobre $\left(\mathrm{CuCl}_{2} \cdot 2 \mathrm{H}_{2} \mathrm{O}\right)$, com
$99 \%$ de pureza, tricloreto de índio $\left(\mathrm{InCl}_{3} \cdot 2 \mathrm{H}_{2} \mathrm{O}\right)$, com $98 \%$ de pureza, e dióxido de selênio $\left(\mathrm{SeO}_{2}\right)$. Os filmes preparados foram submetidos à caracterização por meio das técnicas de difração de raios X, microscopia confocal, transmitância óptica e variação da condutividade elétrica com a temperatura e resistividade elétrica.

As amostras foram caracterizadas pela técnica de difração de raios $X$ utilizando um difratômetro de raios X (DRX), Shimadzu XRD-7000, utilizando os seguintes parâmetros de medida: radiação $\mathrm{CuK \alpha}$ (comprimento de onda de $1,54 \AA$ ), passo em $\theta$ de $0,05^{\circ}$, velocidade de varredura de $1^{\circ} \cdot \mathrm{min}^{-1}$, tempo de contagem de 3,0 s, $2 \theta$ inicial de $20^{\circ}$ e $2 \theta$ final de $80^{\circ}$. A caracterização morfológica baseou-se na análise topográfica dos filmes de CIS depositados sobre os substratos de vidro. Esta análise foi realizada em um microscópio confocal, Olympus OLS 4000, para determinar possíveis trincas e heterogeneidades dos filmes formadas durante o processo de deposição. A espessura dos filmes foi determinada através da análise de suas secções transversais, também por microscopia confocal. A caracterização elétrica foi realizada pela medida da variação da condutividade elétrica em função da temperatura, sendo as medidas de condutividade dos filmes obtidas através do método de dois contatos coplanares, visando a determinação da energia de ativação do processo de condução elétrica dos filmes. Para a medida da resistividade e resistência de folha foi utilizado o método de quatro pontas colineares. A análise de transmitância óptica dos filmes em função do comprimento de onda $(\lambda)$ da radiação incidente (luz) foi realizada visando a determinação dos parâmetros ópticos. O espectrômetro utilizado foi o Spekord M500 UV-Vis, sendo os espectros obtidos na faixa de comprimentos de onda entre 350 e $1100 \mathrm{~nm}$.

\section{RESULTADOS E DISCUSSÃO}

Os arquivos JCDPS 88-0080, 01-077-8902 e 00-0401487 foram utilizados para identificação das fases presentes
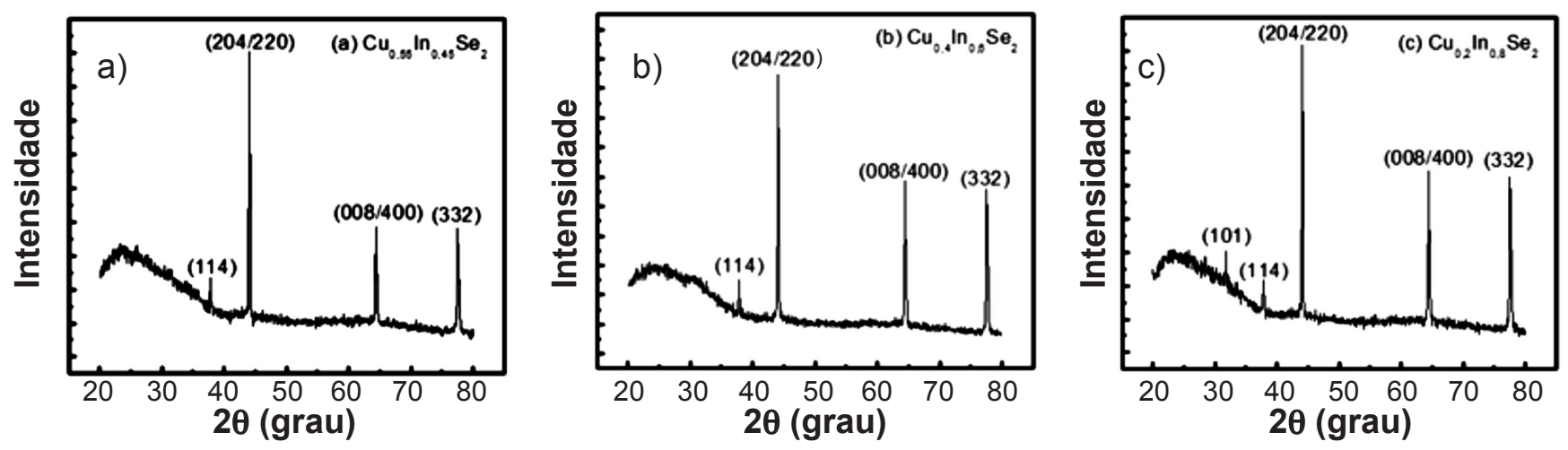

Figura 1: Difratogramas de raios X de filmes de CIS depositados a $400{ }^{\circ} \mathrm{C}$, com fluxo de $1 \mathrm{~mL} / \mathrm{min}$ e tempo de deposição de 10 min, variando a estequiometria, sendo: (a) $\mathrm{Cu}_{0,55} \mathrm{In}_{0,45} \mathrm{Se}_{2}$, (b) $\mathrm{Cu}_{0,4} \mathrm{In}_{0,6} \mathrm{Se}_{2}$ e (c) $\mathrm{Cu}_{0,2} \mathrm{In}_{0,8} \mathrm{Se}_{2}$.

[Figure 1: X-ray diffraction patterns of CIS films deposited at $400{ }^{\circ} \mathrm{C}$ at a flow rate of $1 \mathrm{~mL} / \mathrm{min}$ and deposition time of $10 \mathrm{~min}$, varying the stoichiometry: (a) $\mathrm{Cu} u_{0.55} \mathrm{In}_{0.45} \mathrm{Se} e_{2}$, (b) $\mathrm{Cu} u_{0.4} \mathrm{In}_{0.6} \mathrm{Se} e_{2}$, and (c) $\left.\mathrm{Cu} u_{0.2} \mathrm{In}_{0.8} \mathrm{Se} e_{2}.\right]$ 
nos difratogramas de DRX dos filmes como mostra a Fig. 1. Verificou-se que todas as amostras, independentemente da condição de preparação, foram policristalinas e apresentaram três picos principais em $2 \theta=44,1^{\circ}$ e $64,4^{\circ}$ referentes à fase $\mathrm{CuInSe}_{2}$ e $2 \theta=77,5^{\circ}$ referente à fase $\mathrm{CuSe}$, que corresponderam aos picos de orientação (204)/ (220), (008)/(400) e (332), respectivamente. Os picos em $2 \theta=29,5^{\circ}$ e $37,8^{\circ}$ foram referentes às fases CuSe e $\mathrm{CuSe}_{2}$. O pico referente ao ângulo $2 \theta=29,5^{\circ}$ possuiu menor intensidade para a estequiometria $\mathrm{Cu}_{0.6} \mathrm{In}_{0.4} \mathrm{Se}_{2}$. Já o pico correspondente ao ângulo $2 \theta=37,8^{\circ}$ pôde ser observado em todos os difratogramas, possuindo intensidades próximas para os filmes apresentados na Fig. 1. Pôde-se observar, por meio da Fig. 1, que o pico de maior intensidade indexado com orientação preferencial foi o (204)/(220) referente à fase $\mathrm{CuInSe}_{2}$. Em outros trabalhos, foi relatado que o pico com maior intensidade possuía orientação (112) e o segundo maior pico foi de orientação (204/220) referentes à fase $\mathrm{CuInSe}_{2}$ [4-6], mas também pode-se ter como pico mais intenso de orientação (111) referente à fase CuSe [7, 8]. Em células solares de filmes de disseleneto de cobre e índio, o componente mais importante é a fase CuInSe que funciona como um absorvedor. Para um dispositivo de célula solar eficiente é necessário controlar a estequiometria dos elementos, $\mathrm{Cu}$, In e Se, uma vez que afeta o desempenho das células solares de filmes de $\mathrm{CuInSe}_{2}[9,10]$.

O tamanho dos cristalitos $\left(D_{\text {hkl }}\right)$ foi calculado a partir da fórmula de Scherrer [10] utilizando o pico de maior intensidade, segundo:

$$
\mathrm{D}_{\mathrm{hkl}}=\mathrm{K} \frac{\lambda}{\mathrm{B}_{\mathrm{hkl}} \cdot \cos \left(\theta_{\text {hkl }}\right)}
$$

onde, $\mathrm{K}$ é uma constante que depende da geometria do cristalino (admitido como 0,9 para cristalito esférico), $\lambda$ é o comprimento de onda da radiação incidente $(1,5406 \AA), \mathrm{B}_{\mathrm{hkl}}$ é a largura de pico à meia altura (FWHM) e $\theta$ é o ângulo de difração de Bragg do pico de maior intensidade. Para o filme com estequiometria $\mathrm{Cu}_{0,55} \mathrm{In}_{0,45} \mathrm{Se}_{2}$ obteve-se o valor de 40,5 $\mathrm{nm}$, para estequiometria $\mathrm{Cu}_{0.4} \mathrm{In}_{0.6} \mathrm{Se}_{2} 41,2 \mathrm{~nm}$ e para o filme com estequiometria $\mathrm{Cu}_{0.2} \mathrm{In}_{0.8} \mathrm{Se}_{2} 42,4 \mathrm{~nm}$. Observou-se que o tamanho de cristalito dos filmes praticamente não se alterou para variação da estequiometria e os valores ficaram na faixa de dezenas de nanômetros. Este tamanho é favorável, pois cristalitos menores não são adequados para atuarem na célula solar como camada absorvedora da radiação, devido à elevada densidade de contornos de grão, os quais agem como centros de recombinação dos portadores de carga fotogerados [11]. Os tamanhos de cristalitos estão de acordo com outros relatados na literatura; por exemplo, em [2] os valores encontrados para o tamanho de cristalito variaram de 41 até $57 \mathrm{~nm}$.

Na Fig. 2 são apresentadas as micrografias dos filmes obtidas por microscopia confocal. É visto que as morfologias da superfície de todos os filmes foram semelhantes, independentemente da estequiometria utilizada. As imagens mostraram que de um modo geral as amostras apresentaram-se
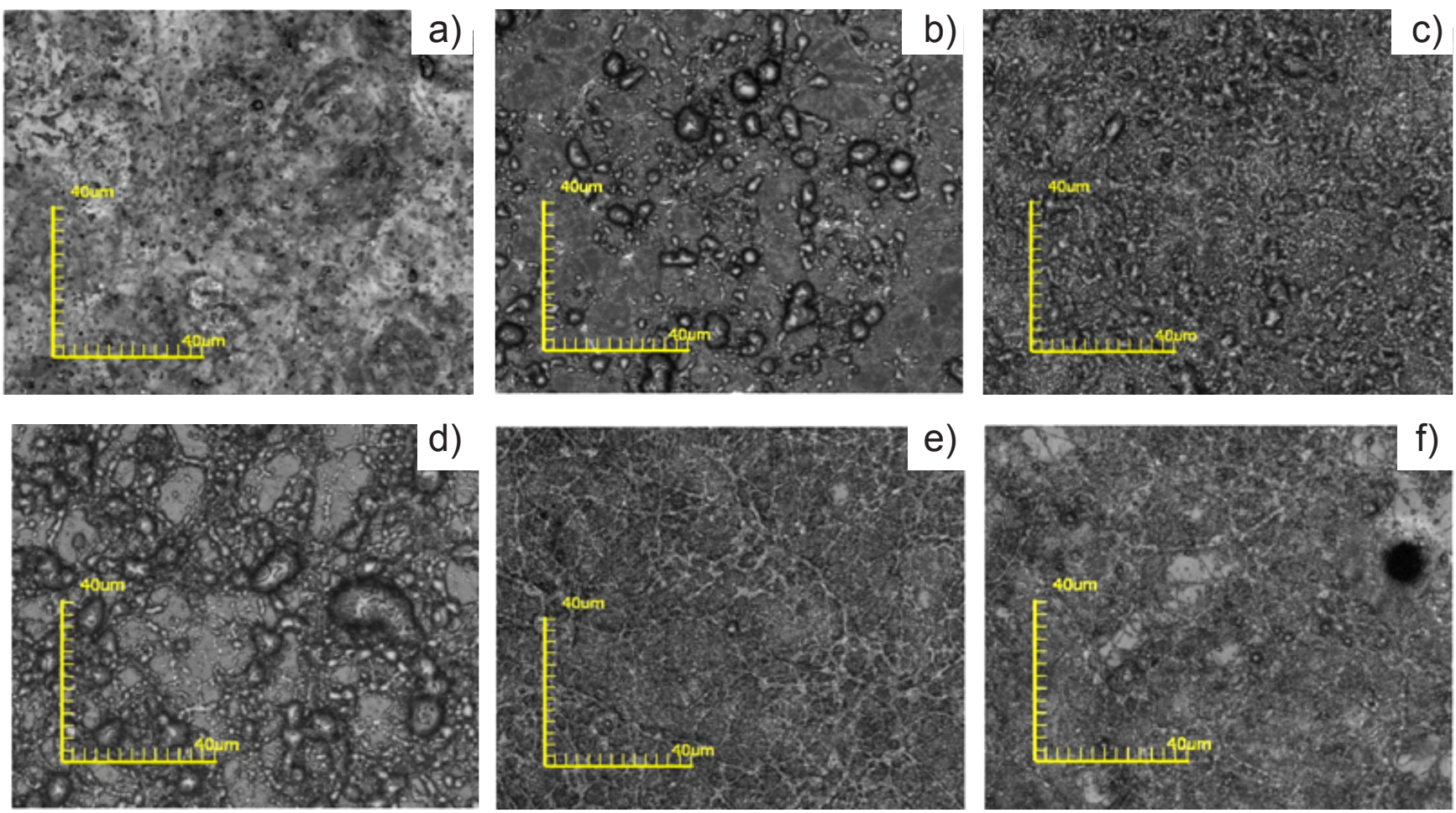

Figura 2: Micrografias de topo obtidas por microscopia confocal mostrando a morfologia da superfície de filmes de CIS depositados com diferentes estequiometrias: (a) $\mathrm{Cu}_{0,55} \mathrm{In}_{0,45} \mathrm{Se}_{2}$, (b) $\mathrm{Cu}_{0,50} \mathrm{In}_{0,50} \mathrm{Se}_{2}$, (c) $\mathrm{Cu}_{0,45} \mathrm{In}_{0,55} \mathrm{Se}_{2}$, (d) $\mathrm{Cu}_{0,40} \mathrm{In}_{0,60} \mathrm{Se}_{2}$, (e) $\mathrm{Cu}_{0,30} \mathrm{In}_{0,70} \mathrm{Se}_{2}$ e (f) $\mathrm{Cu}_{0,20} \mathrm{In}_{0,80} \mathrm{Se}_{2}$. [Figure 2: Confocal microscopy micrographs showing surface morphology of CIS films deposited at varying stoichiometry: (a) Cu $u_{0.5}$ In ${ }_{0.45} S_{2}$, (b) $C u_{0.50} I n_{0.50} S e_{2}$, (c) $C u_{0.45} I n_{0.55} S e_{2},(d) C u_{0.40} I n_{0.60} S e_{2},(e) C u_{0.30} I n_{0.70} S e_{2}$, and (f) $\left.C u_{0.20} I n_{0.80} S e.\right]$ 
Tabela I - Espessura dos filmes de CIS variando a estequiometria.

[Table I - Thickness of CIS films at different stoichiometry.]

\begin{tabular}{cc}
\hline Estequiometria & Espessura do filme $(\mu \mathrm{m})$ \\
\hline $\mathrm{Cu}_{0,55} \mathrm{In}_{0,45} \mathrm{Se}_{2}$ & 1,5 \\
$\mathrm{Cu}_{0,50} \mathrm{In}_{0,50} \mathrm{Se}_{2}$ & 1,8 \\
$\mathrm{Cu}_{0,45} \mathrm{In}_{0,55} \mathrm{Se}_{2}$ & 2,5 \\
$\mathrm{Cu}_{0,40} \mathrm{In}_{0,60} \mathrm{Se}_{2}$ & 2,5 \\
$\mathrm{Cu}_{0,30} \mathrm{In}_{0,70} \mathrm{Se}_{2}$ & 2,6 \\
$\mathrm{Cu}_{0,20} \mathrm{In}_{0,80} \mathrm{Se}_{2}$ & 2,7 \\
\hline
\end{tabular}

sem trincas em toda a superfície e aderentes ao substrato, possuindo superfície com aspecto uniforme. A estrutura tornou-se compacta e densa para filmes com maior concentração de índio. Morfologicamente os filmes foram adequados para serem depositados pela técnica de spraypirólise. As espessuras dos filmes determinadas pela análise de suas seções transversais são mostradas na Tabela I. Para os filmes que possuíram concentração de In maior ou igual a 0,55 , observou-se uma saturação na espessura dos filmes em torno de $2,5 \mu \mathrm{m}$.

São apresentadas na Fig. 3 as curvas de representação linear da variação da condutividade elétrica em função do inverso da temperatura dos filmes de CIS. Os filmes apresentaram curvas características de material semicondutor, já que a condutividade aumentou com o aumento da temperatura de medida. Apresentaram características similares na condutividade elétrica, pois a condutividade teve pequenas alterações com a variação da estequiometria utilizada. Observando o gráfico apresentado

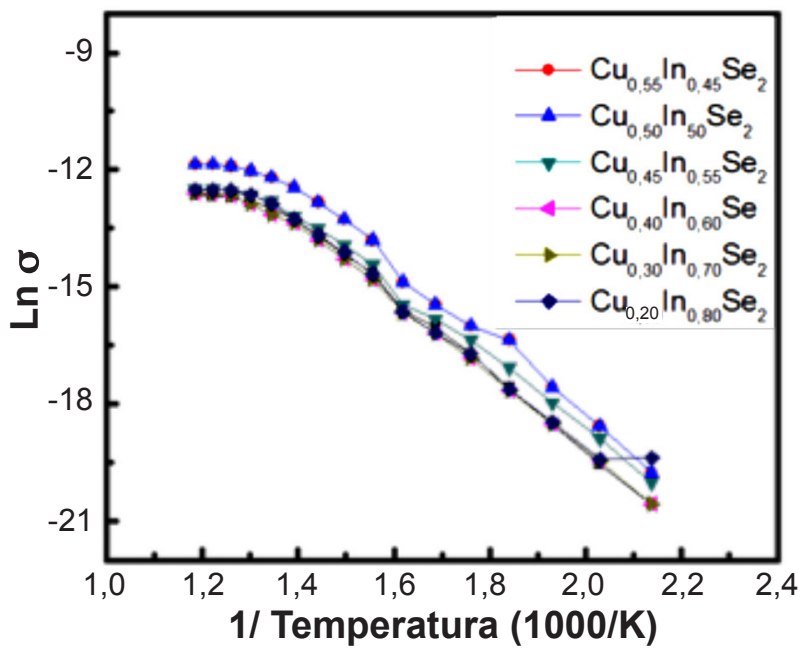

Figura 3: Gráfico da condutividade elétrica em função do inverso da temperatura para filmes de CIS depositados com diferentes estequiometrias.

[Figure 3: Graph of electrical conductivity as a function of reciprocal temperature for CIS films deposited with different stoichiometries.]
Tabela II - Energias de ativação do processo de condução elétrica para filmes de CIS com diferentes estequiometrias.

[Table II - Activation energies for electrical conductivity of CIS films with varying stoichiometry.]

\begin{tabular}{ccc}
\hline Estequiometria & $\begin{array}{c}\text { Energia de } \\
\text { ativação }(\mathrm{eV})\end{array}$ & $\begin{array}{c}\text { Coeficiente de regressão } \\
\text { linear (R) }\end{array}$ \\
\hline $\mathrm{Cu}_{0,55} \mathrm{In}_{0,45} \mathrm{Se}_{2}$ & 0,76 & 0,9960 \\
$\mathrm{Cu}_{0,50} \mathrm{In}_{0,50} \mathrm{Se}_{2}$ & 0,75 & 0,9986 \\
$\mathrm{Cu}_{0,45} \mathrm{In}_{0,55} \mathrm{Se}_{2}$ & 0,74 & 0,9971 \\
$\mathrm{Cu}_{0,40} \mathrm{In}_{0,60} \mathrm{Se}_{2}$ & 0,75 & 0,9991 \\
$\mathrm{Cu}_{0,30} \mathrm{In}_{0,70} \mathrm{Se}_{2}$ & 0,73 & 0,9899 \\
$\mathrm{Cu}_{0,20} \mathrm{In}_{0,80} \mathrm{Se}_{2}$ & 0,72 & 0,9995 \\
\hline
\end{tabular}

na Fig. 3, pôde-se notar uma tendência de região de exaustão ou saturação dos filmes, que representaria a temperatura de transição para o regime de condução intrínseca. Analisando o valor do coeficiente da regressão linear pôde-se comprovar que em todos os filmes o ajuste linear tendeu a 1, como pode ser observado na Tabela II, não sendo por isso necessário o cálculo com duas energias de ativação, uma na faixa de baixa e outra de alta temperatura. Na Tabela II são apresentadas as energias de ativação do processo de condução elétrica dos filmes. Pôde-se observar pouca variação do seu valor com o aumento da concentração de índio presente na solução tendo um valor médio de $0,74 \mathrm{eV}$.

Pelos valores relatados na Tabela III, observa-se que o filme de estequiometria $\mathrm{Cu}_{0,55} \mathrm{In}_{0,45} \mathrm{Se}_{2}$ apresentou maior resistência de folha. Já para os filmes com concentração de In maior que 0,45 ocorreu redução nesse valor, ocorrendo nessa faixa uma flutuação dos valores de resistividade elétrica. Observou-se redução da resistência de folha com o aumento da concentração de índio, sendo o filme de estequiometria $\mathrm{Cu}_{0,20} \mathrm{In}_{0,80} \mathrm{Se}_{2} \mathrm{o}$ de menor valor de resistência de folha e resistividade.

As curvas do coeficiente de absorção $(\alpha)$ versus energia do fóton dos filmes de CIS, onde se variou a concentração de cobre e índio presentes na solução precursora, são apresentadas na Fig. 4. O coeficiente de absorção foi obtido por meio da medida da transmitância através da relação [12]:

Tabela III - Resistência de folha e resistividade elétrica dos filmes de CIS depositados variando a estequiometria.

[Table III - Sheet resistance and resistivity of a CIS films deposited with varying stoichiometry.]

\begin{tabular}{ccc}
\hline Estequiometria & $\begin{array}{c}\text { Resistência de } \\
\text { folha }(\mathrm{M} \Omega / \square)\end{array}$ & $\begin{array}{c}\text { Resistividade } \\
(\mathrm{k} \Omega . \mathrm{m})\end{array}$ \\
\hline $\mathrm{Cu}_{0,55} \mathrm{In}_{0,45} \mathrm{Se}_{2}$ & 90,4 & 0,14 \\
$\mathrm{Cu}_{0,50} \mathrm{In}_{0,50} \mathrm{Se}_{2}$ & 87,3 & 0,16 \\
$\mathrm{Cu}_{0,45} \mathrm{In}_{0,55} \mathrm{Se}_{2}$ & 79,3 & 0,20 \\
$\mathrm{Cu}_{0,40} \mathrm{In}_{0,60} \mathrm{Se}_{2}$ & 71,4 & 0,18 \\
$\mathrm{Cu}_{0,30} \mathrm{In}_{0,70} \mathrm{Se}_{2}$ & 67,7 & 0,17 \\
$\mathrm{Cu}_{0,20} \mathrm{In}_{0,80} \mathrm{Se}_{2}$ & 54,5 & 0,13 \\
\hline
\end{tabular}




$$
\mathrm{I} / \mathrm{Io}=\mathrm{T}=\exp (-\alpha \mathrm{x})
$$

onde, I é a intensidade da radiação incidente que passa pelo filme, Io é a intensidade da radiação emitida, sendo I/Io a transmitância (T), $\alpha$ é o coeficiente de absorção e x a espessura do filme. Trabalhando a expressão a fim de determinar o coeficiente de absorção $(\alpha)$, tem-se:

$$
-\alpha=1 / x(\ln T)
$$

Através dos valores de $\alpha$ (coeficiente de absorção) e utilizando metodologia gráfica proposta por [12], foi traçada a curva de $(\alpha h v)^{2}$ por hv (onde, h é a constante de Planck e $v$ é a frequência), e através da extrapolação da curva foram obtidos os valores dos gaps ópticos dos filmes. Analisando as extrapolações das curvas pôde-se observar redução no valor do gap óptico com o aumento da concentração de índio presente na solução precursora, sendo o menor valor de gap do filme de estequiometria de $\mathrm{Cu}_{0,2} \mathrm{In}_{0,8} \mathrm{Se}_{2}$, com valor de $1,40 \mathrm{eV}$.

Pôde-se inferir pela análise da Fig. 4 que o coeficiente de absorção aumentou para maiores concentrações de índio; isso pode ser atribuído ao fato do maior número de centros absorvedores ter sido gerado com o acréscimo de índio. O filme com maior coeficiente de absorção possuiu estequiometria $\mathrm{Cu}_{0,2} \mathrm{In}_{0,8} \mathrm{Se}_{2}$, tendo como valor $6,8 \times 10^{3}$ $\mathrm{cm}^{-1}$. O fato do coeficiente de absorção não ter alcançado o valor desejado pode estar relacionado ao fato dos filmes do presente estudo não apresentarem o principal pico do difratograma de raios $\mathrm{X}$ apresentado na literatura, sendo este (112) [9, 10]. Também pode estar relacionado com a

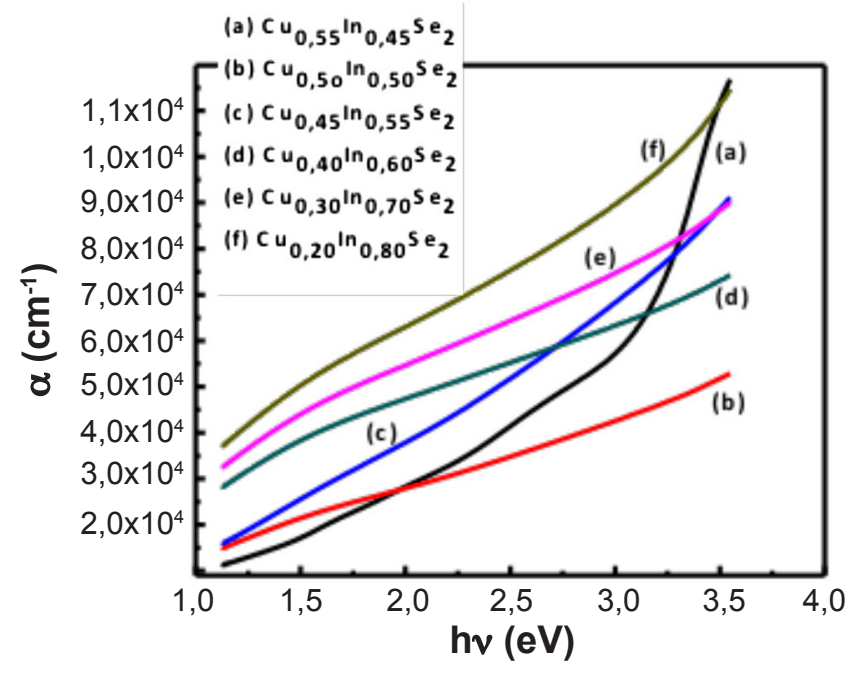

Figura 4: Gráfico de coeficiente de absorção ( $\alpha$ ) versus energia (hv) dos filmes de CIS depositados com fluxo de $1 \mathrm{~mL} / \mathrm{min}$, temperatura de $400{ }^{\circ} \mathrm{C}$, tempo de 10 min e variando a concentração de cobre e índio.

[Figure 4: Graph of absorption coefficient ( $\alpha$ ) versus energy ( $h v$ ) of CIS films deposited with flow of $1 \mathrm{~mL} / \mathrm{min}$, temperature of $400{ }^{\circ} \mathrm{C}$, time of 10 min and varying the concentration of copper and indium.] reflectância, que nos cálculos realizados para obtenção do coeficiente de absorção esta não foi considerada, sendo este fato relevante, já que os filmes se apresentaram com um aspecto espelhado, principalmente os que possuíram maior concentração de cobre.

\section{CONCLUSÕES}

Filmes de disseleneto de cobre e índio foram depositados sobre substratos de vidro, pela técnica de spray-pirólise, variando sua estequiometria com o objetivo de serem aplicados como camada absorvedora de células solares. Por meio das caracterizações foi possível estabelecer que o filme de estequiometria $\mathrm{Cu}_{0,2} \mathrm{In}_{0,8} \mathrm{Se}_{2}$, com parâmetros fixos de deposição de $400{ }^{\circ} \mathrm{C}$ de temperatura de substrato, fluxo da solução precursora de $1 \mathrm{~mL} / \mathrm{min}$ e tempo de deposição de $10 \mathrm{~min}$, teve aspecto contínuo e sem trincas, sendo esta a condição mais propícia para a deposição de filmes de disseleneto de cobre e índio sobre substrato de vidro utilizando a técnica spray-pirólise para aplicação como camada absorvedora de células fotovoltaicas, resultando em coeficiente de absorção de $6,8 \times 10^{3} \mathrm{~cm}^{-1}$ e menor resistividade elétrica de $0,13 \mathrm{k} \Omega . \mathrm{m}$ em temperatura ambiente.

\section{AGRADECIMENTOS}

À CAPES pela bolsa de doutoramento e ao CNPQ pelo apoio financeiro.

\section{REFERÊNCIAS}

[1] A.A. Alaa, H.H. Afify, Mater. Res. Bull. 43 (2008) 15391548 .

[2] L.P. Desmukh, R.V. Suryawanshi, E.U. Masumdar, M. Sharon, Sol. Energy 86 (2012)1910-1919.

[3] C.R. Kim, Y. H. Seung, H. Chih, T. J. L. Chang, Curr. Appl. Phys. 10 (2010) 383-386.

[4] Y. Li, S.S. Shaikh, S. Menezes, Thin Solid Films 524 (2012) 20-25.

[5] F.A. Mahmoud, M. Boshta, M.H. Sayed, Mater. Electron 24 (2013) 448-451.

[6] A.P. Samantilleke, M. Sahal, L. Ortiz, M.F. Cerqueira, B. Marí, Thin Solid Films 519 (2011) 7272-7275.

[7] N.M. Shah, J.R. Ray, K.J. Patel, Thin Solid Films 517 (2009) 3639-3644.

[8] Z. Han, D. Zhang, Q. Chen, T. Mei, S. Zhuang, Powder Technol. 249 (2013) 119-125.

[9] S.R. Kodigala, Thin Film Solar Cells 35 (2010) 115-194. [10] C.J. Panchal, V.R. Kheraj, J.R. Ray, M.S. Desai, Sol. Energy 83 (2009) 753-760.

[11] L.R. Cruz, Q.G.S San Miguel, Matéria 17 (2012) 961972.

[12] J. Tauc, A. Menth, J. Non-Cryst. Solids 8, 10 (1972) $569-585$.

(Rec. 05/04/2016, Rev. 23/06/2016, 01/08/2016, Ac. 23/10/2016) 